\title{
Utility of Hippocrates' prognostic aphorism to predict death in the modern era: prospective cohort study
}

\author{
@) (ब) OPEN ACCESS
}

\author{
Philip D St John associate professor ${ }^{1}$, Patrick R Montgomery consultant geriatrician ${ }^{2}$
}

${ }^{1}$ Section of Geriatric Medicine, Department of Medicine, and the Centre on Aging, University of Manitoba, GE 547 Health Sciences Centre, 820 Sherbrook St, Winnipeg, MB, Canada R3A 1R9; ${ }^{2}$ Vancouver Island Health Authority Geriatric Services Memorial Pavilion, Victoria, British Columbia, Canada

\begin{abstract}
Objective To determine if one of Hippocrates' aphorisms, identifying good cognition and good appetite as two prognostic factors, predicts death in community living older adults in the modern era.

Design Secondary analysis of an existing population based cohort study. Setting Manitoba Study of Health and Aging.

Participants 1751 community living adults aged more than 65 enrolled in the Manitoba Study of Health and Aging in 1991 and followed over five years.

Main outcome measure Time to death.

Methods We recreated the hippocratic prognosticator using an item that measures appetite drawn from the Center for Epidemiologic Studies-depression subscale, and the mini-mental state examination, with a score of $>25$ being considered as normal. People with normal cognition and appetite were compared with those with either poor cognition or poor appetite. We constructed Cox regression models, adjusted for age, sex, education, and functional status.

Results The prognostic aphorism predicted death, with an unadjusted hazard ratio of 2.37 ( $95 \%$ confidence interval 1.93 to 2.88 ) and a hazard ratio of 1.71 (1.37 to 2.12) adjusted for age, sex, and education. Both poor appetite and poor cognition predicted death. The sensitivity and specificity were not, however, sufficient for the measure to be used alone.

Conclusion An aphorism devised by Hippocrates millennia ago can predict death in the modern era.
\end{abstract}

\section{Introduction}

The notion that disease is based on natural causes, the importance of bedside medicine, and many current ethical principles all have their roots in the hippocratic tradition. Diagnostic skill was important, ${ }^{1}$ but accurate prognostication was more highly prized: "The best physician was one who could prevent and predict." ${ }^{2}$ Prognostication went beyond simply forecasting the future, to encompass a broader understanding of the cause, context, and clinical course of an individual's disease. ${ }^{34}$ Many of Hippocrate's aphorisms were related to prognostic factors.

Aphorisms are short, pithy statements summarising an observation or giving a recommendation..$^{5}$ One of these aphorisms stated: "It augurs well, if the patient's mind is sound, and he accepts all food that's offered him; but, if the contrary conditions do prevail, the chances of recovery are slim." Numerous, differing translations of this aphorism exist, but all contain two key prognostic factors: good appetite and good cognition. There is evidence for death being predicted by cognitive impairment ${ }^{78}$ and weight loss. ${ }^{9}{ }^{10}$ However, no study has been done to ascertain if the original hippocratic aphorism accurately predicts outcomes.

We determined if Hippocrates' aphorism could predict death in community living older adults in the modern era and if a gradient effect existed, with more severe appetite and cognitive loss being associated with a worse prognosis than less severe appetite and cognitive loss.

\section{Methods}

\section{Sample}

We used data from the Manitoba Study of Health and Aging (MSHA), a prospective cohort study conducted in conjunction with the Canadian Study of Health and Aging. ${ }^{11}$ In that study community living adults aged more than 65 were randomly selected from a representative population registry. The original survey was conducted in 1991-92, and follow-up was carried 
out in 1996-97. Overall, 2890 people were initially selected. Of these, 443 refused to participate, 480 were not eligible (died, entered nursing home, or too ill), 162 could not be located, and 54 did not complete the screening questionnaire. At least one data source provided complete follow-up for all participants for mortality over the five years.

\section{Outcome measures}

Participants self reported age, sex, and education. We used an item from the Center for Epidemiologic Studies-depression subscale to assess appetite ${ }^{12}$ : "I did not feel like eating; my appetite was poor." We scored this item: 0 , rarely or none of the time ( $<1$ day); 1 , some or a little of the time (1-2 days); 2 , occasionally or a moderate amount of the time (3-4 days), and 3 , most of the time (5-6 days). We measured cognition using the modified mini-mental state examination $(3 \mathrm{MS}),{ }^{13}$ an expanded version of the mini-mental state examination,,${ }^{14}$ and functional status using the older Americans resources and services scale. ${ }^{15}$ On the basis of the results we grouped cognition into no disability, mild disability, and moderate, severe, or total disability.

\section{Aphorism prognosticator}

Based on the hippocratic aphorism we created a dichotomous prognostic indicator. We considered participants with a good prognosis to be those with a mini-mental state examination score of greater than 25 and who had a poor appetite rarely or none of the time (that is, 0 on the depression subscale item). We considered participants with a poor prognosis to be either those with a mini-mental state examination score of 25 or less, or those who had a poor appetite some of the time, occasionally, or most of the time.

\section{Risk index}

Since the effect of cognition ${ }^{16}$ and appetite are gradient effects rather than threshold effects, we constructed a continuous risk score (table $1 \Downarrow$ ). For this, we simply assigned a score of 0 to 3 for categories of the mini-mental state examination arbitrarily chosen before we conducted the analysis, and a score of 0 to 3 for the Center for Epidemiologic Studies-depression subscale. This gave a score from 0 to 6 .

\section{Outcomes}

The primary outcome was time to death. We obtained information on death over the five year interval by proxy reports, death certificates, and administrative records. Mortality was coded as either alive or dead by the end of the Manitoba Study of Health and Aging-2. We used the methods of the Canadian Study of Health and Aging to determine the time to death. ${ }^{17}$ Briefly, we used the date of death on the death certificate $(n=247)$, and if these data were not available, we obtained the date of death from proxy report $(\mathrm{n}=73)$ or administrative data $(n=97)$. We considered the participants to have been censored after the end of the second phase of follow-up.

\section{Statistical analysis}

For bivariate analyses, we used $\chi^{2}$ tests for categorical variables. For continuous variables, we used Student's $t$ tests (assuming unequal variance). We constructed Kaplan-Meier plots and Cox regression models. The primary variable of interest was the hippocratic aphorism, which was a dichotomous variable (good prognosis versus poor prognosis). Potential confounding factors were age (years), sex, education (years of formal education), and functional status (considered as no impairment, mild impairment, and moderate or severe impairment). We also conducted a sensitivity analysis using the continuous risk index, and considered several cut-off points for the mini-mental state examination (see supplementary file). We determined the sensitivity and specificity of the aphorism. Finally, we constructed receiver operating characteristic curves for the risk index using the outcome of death and used a c statistic to calculate the area under the receiver operating characteristic curve.

\section{Results}

Table $2 \Downarrow$ shows the characteristics of the 1751 participants. The simple aphorism predicted mortality (figure $\Downarrow$ ). The effect was attenuated by considering other factors but remained significant (table $3 \Downarrow$ ). In addition, each item predicted death: the unadjusted hazard ratio for appetite was 1.79 (95\% confidence interval 1.44 to 2.22) and the hazard ratio adjusted for age, sex, education, and functional status was 1.63 (1.31 to 2.03 ). The unadjusted hazard ratio for cognitive impairment was 2.21 (1.82 to 2.68) and the adjusted hazard ratio was 1.46 (1.16 to 1.83 ). The continuous risk index predicted survival in Cox regression analyses: the unadjusted hazard ratio was 0.74 ( 0.70 to 0.79 ) per point on the score and the adjusted hazard ratio was 0.87 (0.80 to 0.94$)$.

When considering the aphorism as a diagnostic test for predicting death, the sensitivity was $65 \%$ and the specificity was $60 \%$. The continuous risk index predicted death, with an area under the receiver operating characteristic of $0.65(95 \%$ confidence interval 0.62 to 0.68 , see supplementary file). The area under the receiver operating characteristic curve for the four point appetite item was 0.56 ( 0.53 to 0.58$)$ and for cognition was 0.63 (0.60 to 0.66$)$.

\section{Discussion}

Accurate prognostication was highly prized in the hippocratic tradition. ${ }^{14}$ Recently, attention has returned to prognostic models, ${ }^{17}$ but no one has examined the hippocratic aphorism in the context of a modern dataset. We found that people with a poor prognosis according to this aphorism had a mortality rate that was twice as high as those with a good prognosis. Furthermore, considering cognition and appetite as continuous factors adds prognostic information, by expanding the range across which the increased risk is apparent. Each factor predicted death, with cognition associated with more predictive accuracy than appetite. However, the sensitivity and specificity of these factors do not allow ascertainment of the risk of death with certainty. Nevertheless, they are likely to be accurate enough to alert doctors to patients who may do poorly in the future. We also found that age, sex, and functional status all predicted adverse outcomes, and including them in statistical models attenuated the effect of the prognostic score. Incorporating these factors into a more complete risk index could improve the accuracy of the measure.

\section{Strengths and weaknesses of this study}

Our approach has both strengths and limitations. Firstly, the exact wording of the original aphorism is not known. The hippocratic corpus was probably not written by Hippocrates, but rather by several authors, and was modified and translated over time. ${ }^{1}$ Furthermore, there are many prognostic aphorisms, but we have considered only one. Thus it is impossible to replicate all hippocratic prognostic factors exactly. Secondly, 
the dataset we used is somewhat ancient in itself, having been generated in the 1990s. Thirdly, we used measures that were not available to Hippocrates and we arbitrarily chose cut-off points for the mini-mental state examination. Fourthly, we used data from a simple screening questionnaire (not from the clinical examination that followed). Data in Hippocrates' time would have been gathered by the physicians themselves, which could increase, or perhaps decrease, the reliability of the measures. Finally, neither we nor Hippocrates followed the guidelines for developing and validating a clinical prediction rule. We simply considered the original hippocratic observations as the derivation dataset and proceeded directly to a validation cohort. Of course, Hippocrates antedated modern statistical methods and procedures for devising risk indices by several thousand years, so he was somewhat at a disadvantage.

The strengths of our study were that the Manitoba Study of Health and Aging was a large sample drawn from a representative sampling frame, and the aphorism could be replicated fairly accurately.

\section{Conclusions}

We do not advocate using this exact measure in clinical practice. However, we feel that several important lessons can be learnt. Firstly, the critical role of cognition in prognostication is often overlooked. Many centuries have elapsed between Hippocrates' existence and now, during which this observation has been little translated into clinical care. At some points, risk scores have incorporated cognition, ${ }^{18}$ but many case mix adjustment tools and prognostic indices still do not incorporate cognitive measures. It is possibly time to develop risk assessment tools that consider cognition.

Secondly, accurate prognostication is as important today as it was in Hippocrates' time. The current haphazard application of highly technical and expensive treatments to all people regardless of treatment goals, comorbidity, cognition, or functional status is a prominent feature of modern medicine. Identifying those who may benefit from aggressive care will be important in the future, and lessons may be learnt from the past.

Thirdly, the importance of simple clinical measures remains relevant today. Hippocrates' aphorism is brief and pithy, yet contains important data. Simple global measures, such as cognition and appetite, are easily measured, reproducible, and important. The hippocratic tradition repeatedly stressed the importance of symptoms and clinical examination. With our current focus on advanced diagnostic tests we may omit important data that are easily obtained during history taking and physical examination. Even in our time, it may be worth listening to, and examining, patients.

Contributors: Both authors contributed to data collection at various points in the study, and to the drafting of the manuscript. PDStJ conducted the analyses and is the guarantor.

Funding: The Manitoba Study of Health and Aging was funded primarily by Manitoba Health, with additional funding provided through the Canadian Study of Health and Aging by the seniors' independence research program of the National Health Research and Development Program of Health Canada. No endorsement from these agencies is implied. The sponsors had no role in the design, methods, subject recruitment, data collection, analysis, or preparation of the paper. Competing interests: All authors have completed the ICMJE uniform disclosure form at www.icmje.org/coi_disclosure.pdf and declare: no support from any organisation for the submitted work; no financial relationships with any organisations that might have an interest in the submitted work in the previous three years; no other relationships or activities that could appear to have influenced the submitted work.

Ethical approval: All participants or the appropriate proxy gave informed consent for participation, and the Manitoba Study of Health and Aging received ethical approval from the human research ethics board of the Faculty of Medicine of the University of Manitoba.

Data sharing: the technical appendix and anonymised individual level data used for these analyses are available from the corresponding author at pstjohn@hsc.mb.ca.

Transparency: The lead author (PDStJ) affirms that this manuscript is an honest, accurate, and transparent account of the study being reported; that no important aspects of the study have been omitted; and that any discrepancies from the study as planned (and, if relevant, registered) have been explained.

1 Porter R. The greatest benefit to mankind: a medical history of humanity. WW Norton, 1997.

2 Marketos SG, Skiadas PK. The modern hippocratic tradition. Some messages for contemporary medicine. Spine 1999;24:1159-63.

3 Dachez R. Histoire de la medicine, de l'antiquite au XXe siecle. Tallandier, 2004

4 Brock A. Greek medicine. Dent, 1929.

5 Chandler ET, Bloomfield RL. Aphorisms: wisdom for all seasons. South Med J 1987;80:84-9.

6 Scholtz M. Hippocrates' aphorisms. Cal West Med 1940;53:35.

7 Todd S, Barr S, Roberts M, Passmore AP. Survival in dementia and predictors of mortality: a review. Int J Geriatr Psychiatry 2013;28:1109-24.

8 Witlox J, Eurelings LS, de Jonghe JF, Kalisvaart KJ, Eikelenboom P, van Gool WA Delirium in elderly patients and the risk of postdischarge mortality, institutionalization, and dementia: a meta-analysis. JAMA 2010;304:443-51.

9 Newman AB, Yanez D, Harris T, Duxbury A, Enright PL, Fried LP. Weight change in old age and its association with mortality. J Am Geriatr Soc 2001;49:1309-18.

10 Donini LM, Savina C, Cannella C. Eating habits and appetite control in the elderly: the anorexia of aging. Int Psychogeriatr 2003;15:73-87.

11 Canadian study of health and aging: study methods and prevalence of dementia. Can Med Assoc J 1994;150:899-913.

12 Radloff $L$. The CES-D scale: a self-report depression scale for research in the general population. Appl Psychol Meas 1977;1:385-401.

13 Teng EL, Chui HC. The Modified Mini-Mental State (3MS) examination. J Clin Psychiatry 1987;48:314-8.

14 Folstein MF, Folstein SE, McHugh PR. "Mini-mental state". A practical method for grading the cognitive state of patients for the clinician. J Psychiatr Res 1975;12:189-98.

15 Fillenbaum GG. Multidimensional functional assessment of older adults: the Duke Older Americans Resources and Services Procedures. Lawrence Erlbaum, 1988.

16 St John PD, Montgomery PR, Kristjansson B, McDowell I. Cognitive scores, even within the normal range, predict death and institutionalization. Age Ageing 2002;31:373-8.

17 Dubois MF, Hebert R. Imputation of missing dates of death or institutionalization for time-to-event analyses in the Canadian Study of Health and Aging. Int Psychogeriatr 2001:13(Suppl 1):91-7.

18 Arnold J, Exton-Smith AN. The geriatric department and the community value of hospital treatment in the elderly. Lancet 1962;280:551-3.

\section{Accepted: 14 November 2014}

\section{Cite this as: BMJ 2014;349:97390}

This is an Open Access article distributed in accordance with the Creative Commons Attribution Non Commercial (CC BY-NC 4.0) license, which permits others to distribute, remix, adapt, build upon this work non-commercially, and license their derivative works on different terms, provided the original work is properly cited and the use is non-commercial. See: http://creativecommons.org/licenses/by-nc/4.0/. 


\section{What is already known on this topic}

One of the hippocratic aphorisms on prognosis stated that good appetite and good cognition were favourable prognostic factors Poor cognition and weight loss are known to predict mortality, but the original aphorism has never been validated

\section{What this study adds}

Poor cognition and poor appetite in community living older adults predict mortality, and the hippocratic aphorism itself predicts mortality The aphorism is not accurate enough to predict with certainty who will die, but the findings highlight the importance of simple global measures of health

\section{Tables}

\section{Table 1| Risk score based on Hippocratic aphorism, with higher scores indicating a more favourable prognosis}

\begin{tabular}{lcc} 
Item & No of participants & Score \\
\hline Mini-mental state examination score: & & \\
\hline$<20$ & 86 & 0 \\
\hline $20-23$ & 211 & 1 \\
\hline $24-26$ & 429 & 2 \\
\hline$>26$ & 1025 & 3 \\
\hline Poor appetite: & & \\
\hline Rarely or none of time (<1 day) & 1436 & 3 \\
\hline Some or little of time (1-2 days) & 155 & 2 \\
\hline Occasionally or moderate amount of time (3-4 days) & 70 & 1 \\
\hline Most of time (5-6 days) & 90 & 0 \\
\hline
\end{tabular}


Table 2| Baseline characteristics of sample at baseline and mortality over five years. Values are numbers (percentages) unless stated otherwise

\begin{tabular}{|c|c|c|c|}
\hline Characteristics & Poor prognosis† $(n=719)$ & Good prognosis $¥(n=1032)$ & Total sample $(n=1751)$ \\
\hline Mean (SD) age (years) & $79.9(7.5)^{\star}$ & $75.9(6.4)^{*}$ & $77.5(7.1)$ \\
\hline Women & $396(55)^{*}$ & $629(61)^{*}$ & $1025(58)$ \\
\hline Mean (SD) education (years) & $7.8(3.7)^{\star}$ & $10.3(3.1)^{*}$ & $9.3(3.6)$ \\
\hline \multicolumn{4}{|l|}{ Functional status: } \\
\hline No impairment & $345(48)^{*}$ & $742(72)^{*}$ & $1087(62)$ \\
\hline Mild or moderate impairment & $230(32)^{*}$ & $220(21)^{*}$ & $450(25)$ \\
\hline Severe impairment & $144(20)^{*}$ & $70(7)^{*}$ & $214(12)$ \\
\hline Mortality (\% dead) & $252(35)^{*}$ & $165(16)^{*}$ & $417(24)$ \\
\hline
\end{tabular}

${ }^{*} \mathrm{P}<0.05$.

†Mini-mental state examination score $\leq 26$, or a poor appetite some of time, occasionally, or most of time. ¥Mini-mental state examination score $>25$ and a poor appetite rarely or none of time. 
Table 3| Results of Cox regression models for dichotomous prognosticator derived from aphorism

\begin{tabular}{lccc} 
& \multicolumn{3}{c}{ Hazard ratio $(95 \%$ Cl) for mortality } \\
\cline { 2 - 4 } Variables & Unadjusted model 1 & Adjusted model 2* & Model 3: full model \\
Prognosis (reference good) & $2.37(1.93$ to 2.88$)$ & $1.71(1.37$ to 2.12$)$ & $1.50(1.19$ to 1.86$)$ \\
\hline Age (per year) & - & $1.07(1.06$ to 1.08$)$ & $1.06(1.04$ to 1.07$)$ \\
\hline Sex (reference male) & - & $0.58(0.47$ to 0.70$)$ & $0.51(0.41$ to 0.62$)$ \\
\hline Education (per year) & - & $1.00(0.97$ to 1.02$)$ & $1.00(0.97$ to 1.02$)$ \\
\hline Functional status (reference no impairment) & - & - & - \\
\hline Mild or moderate impairment & - & - & $1.80(1.41$ to 2.29$)$ \\
\hline Severe impairment & - & - & $2.62(2.01$ to 3.43$)$ \\
\hline
\end{tabular}

*Adjusted for age, sex, and education. 


\section{Figure}

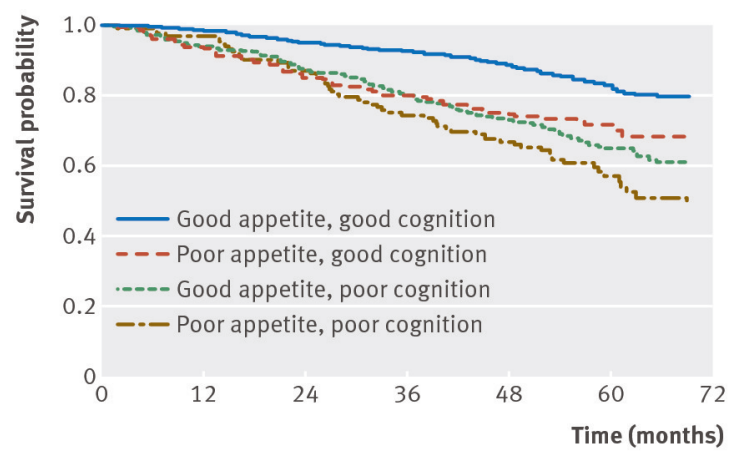

Kaplan-Meier survival curve of older community living adults based on appetite and cognition 